\title{
Ordered magnesium-lithium alloys: First-principles predictions
}

\author{
Richard H. Taylor \\ Department of Physics and Astronomy, Brigham Young University, Provo, Utah 84602, USA
}

\begin{abstract}
Stefano Curtarolo
Department of Mechanical Engineering and Materials Science and Department of Physics, Duke University, Durham, North Carolina 27708, USA

and Department of Materials and Interfaces, Weizmann Institute of Science, Rehovoth 76100, Israel
\end{abstract}

Gus L. W. Hart*

Department of Physics and Astronomy, Brigham Young University, Provo, Utah 84602, USA

(Received 16 October 2009; revised manuscript received 3 January 2010; published 26 January 2010)

\begin{abstract}
Magnesium-lithium (Mg-Li) alloys are among the lightest structural materials. Although considerable work has been done on the Mg-Li system, little is known regarding potential ordered phases. A first and rapid analysis of the system with the high-throughput method reveals an unexpected wealth of potentially stable low-temperature phases. Subsequent cluster expansions constructed for bcc and hcp superstructures extend the analysis and verify our high-throughput results. Of particular interest are those structures with greater than 13 at. \% lithium, as they exhibit either partial or complete formation as a cubic structure. Order-disorder transition temperatures are predicted by Monte Carlo simulations to be in the range 200-500 K.
\end{abstract}

DOI: $10.1103 /$ PhysRevB.81.024112

PACS number(s): $61.50 . \mathrm{Ks}, 81.30 . \mathrm{Hd}$

\section{MOTIVATION AND BACKGROUND}

Emerging technologies increasingly depend on the production of ultralight-weight materials. Magnesium-lithium (Mg-Li) alloys are the lightest metallic alloys, having densities near that of plastics, ${ }^{1-3}$ and are strong enough to be used in a variety of high-performance applications. In particular, $\mathrm{Mg}-\mathrm{Li}$ alloys are good candidates for material applications in industries such as aerospace and automotive manufacturing. ${ }^{4}$

As an alloying agent in magnesium, lithium is advantageous principally because of its low density and cubic crystal structure. The addition of lithium converts the hexagonalclose-packed (hcp) structure of natural magnesium to a more ductile and formable body-centered-cubic (bcc) alloy. Addition of 13 at. \% Li partially converts the alloy to a cubic structure and content exceeding 33 at. \% results in total conversion. ${ }^{5}$

Although the Mg-Li system has been studied extensively, ${ }^{5-11}$ evidence suggesting the formation of ordered phases is notably sparse. Diffusionless transformations of the martensitic type have been observed in pure $\mathrm{Li}$, and similar transformations occur in low-temperature magnesium alloyed lithium at certain concentrations. ${ }^{11,12}$ Binary ordered phases have not been conclusively identified, however, and any instances contained in the literature are indeterminate. ${ }^{6,9,13,14}$

The results of high-throughput (HT) ab initio calculations performed with the AFLOW package (described later) show, however, that the heat of formation is negative for a large number of potential structure types, implying the existence of at least one thermodynamically stable ordered phase (Fig. 1).

\section{FIRST-PRINCIPLES METHOD}

Following the HT results, we have made predictions of $\mathrm{Mg}-\mathrm{Li}$ ordered phases using the cluster-expansion (CE) method - a first-principles-based approach in which input data is mapped to a truncated Ising-type Hamiltonian. The configurational Hamiltonian allows for a fast ground-state search (GSS) over many derivative superstructures. ${ }^{16}$ The $\mathrm{CE}$ method was applied to the $\mathrm{Mg}$ and $\mathrm{Li}$ lattice configurations (bcc and hcp, respectively). Although minimum-energy configurations may exist outside those considered, superstructures derived from the parent lattices of the alloy constituents generally include the lowest-energy configurations. This assumption is particularly supported for $\mathrm{Mg}-\mathrm{Li}$ by the existence of bcc derivative superstructures exclusively as ground states in the HT results and by phases present in experimental phase diagrams (Fig. 2).

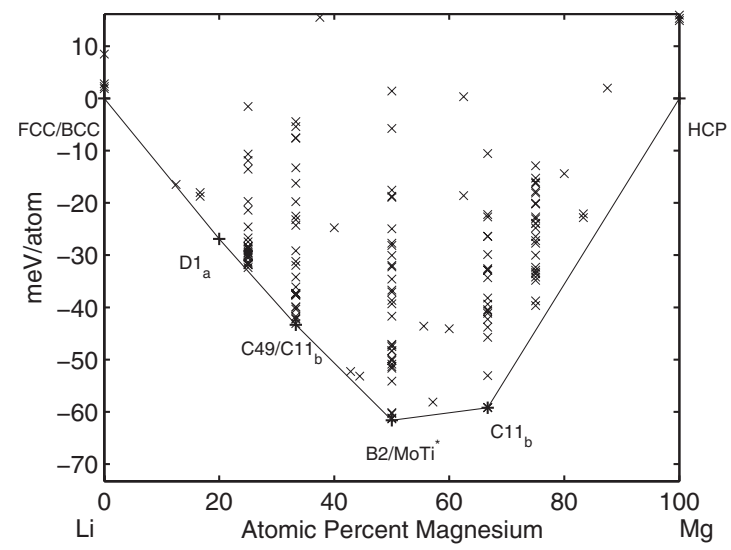

FIG. 1. High-throughput formation enthalpy calculations for $\mathrm{Mg}$-Li. Structures resting on the convex hull are labeled according to their Strukturbericht designation and are displayed prominently. Note that many computed structures have negative formation enthalpy, suggesting an ordering system. MoTi* prototype from Ref. 15 . 


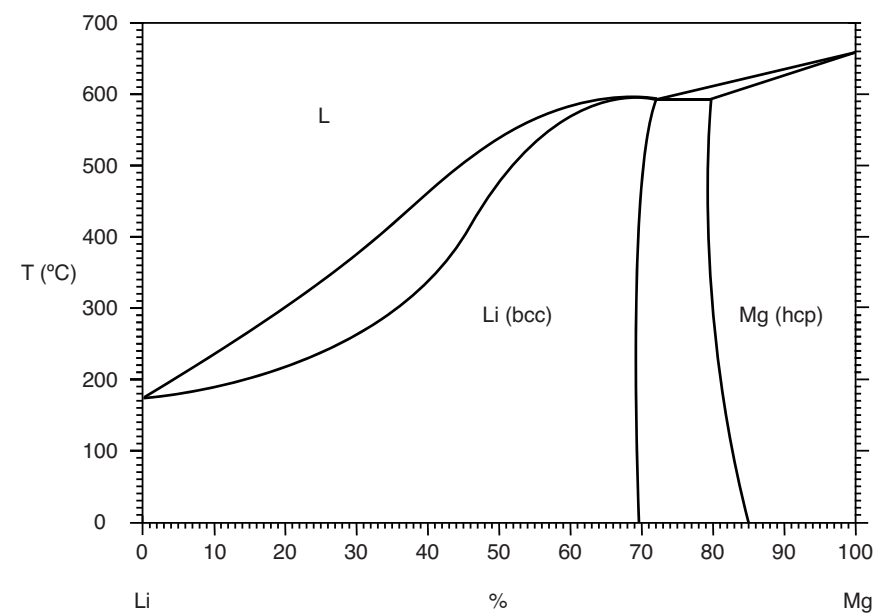

FIG. 2. Schematic LiMg phase diagram constructed after Refs. 8 and $17-21$.

Quantum-mechanical energies used for the HT and CE approaches were computed using density-functional theory (DFT) as implemented by the Vienna $a b$ initio simulation package (VASP) software. ${ }^{22}$ Following the method of Froyen, ${ }^{23}$ so-called "equivalent points" were used to form the k-point mesh, resulting in reduced systematic error in the calculation of formation enthalpy. Perdew-Burke-Ernzerhof projector-augmented wave pseudopotentials with semicore $s$ and $p$ states treated as valence were used. ${ }^{24,25}$ The plainwave energy cutoff was approximately $339 \mathrm{eV}$. Energies were calculated at zero temperature and pressure without spin polarization, zero-point motion, or lattice vibrations. All crystal structures were fully relaxed (cell volume and shape and the basis-atom coordinates inside the cell). Numerical convergence to about $1 \mathrm{meV} /$ atom was ensured by the highenergy cutoff and by dense k-point meshes.

In the HT work, the calculations were performed using the high-throughput framework AFLOw. ${ }^{15,26-29}$ For each system, the energies of 204 crystal structures were calculated. In addition to the 176 configurations described in Ref. 15, these included all the symmetrically distinct hcp-based, bcc-based, and fcc-based superstructures ${ }^{30,31}$ with up to four atoms per cell and additional prototypes. ${ }^{32}$ The additional prototypes were considered because they are common or related to $\mathrm{Mg}$ alloys. ${ }^{33,34}$ This protocol gives reasonable results. In Ref. 15, it was shown that the probability of reproducing the correct ground state, if well defined and not ambiguous, is $\eta_{c}^{\star} \sim 96.7 \%$ [ "reliability of the method," Eq. (3)]. For the HT approach, we did not consider lattice superstructures with more than four atoms per cell due to the fact that their number increases exponentially. ${ }^{35}$ Therefore, even if it is impossible to rule out the existence of an undetected ground state, the protocol is expected to give a reasonable balance between high-throughput speed and scientific accuracy to determine miscibility of $\mathrm{Mg}-\mathrm{Li}$ alloys.

\section{CLUSTER-EXPANSION METHOD AND GROUND- STATE SEARCH RESULTS}

In the CE formalism, atomic configuration is defined by first associating a set of "spin" values to atomic type. The configurational properties of a structure are then captured by the averages of spin products or values of correlation functions on the lattice.

The correlation functions, evaluated over each symmetrically unique "cluster," form a mathematically complete basis for a lattice of size $N$ by which any configurational property may be expanded. ${ }^{36} \mathrm{~A}$ set of constants termed effective cluster interactions (ECIs) form the coefficients of the expansion by which the material property in question is expressed.

The utility of the CE method is that a physical quantity such as energy may be expanded entirely in configuration space. This allows for exhaustive searches over configurational domains in order, for example, to find minima. Given cluster-expanded formation enthalpy, minimum-energy structures serve as strong candidates for thermodynamically stable ordered states. In the case of a binary system, the CE expresses the energy of a lattice configuration $\sigma$ as

$$
E_{\mathrm{CE}}(\sigma)=J_{0}+\sum_{f} \sum_{1}^{N_{f}} \Pi_{f}(\sigma) J_{f},
$$

where $\sigma$ defines the occupation of $N$ lattice sites by A and B atoms, $J_{f}$ is the ECI for cluster $f, N_{f}$ is the number of clusters of type $f$, and $\Pi_{f}$ are the averaged spin ( \pm 1 for binary systems) products for cluster $f$ in configuration $\sigma$.

Effective cluster interactions were found using leastsquares regression and a genetic algorithm. ${ }^{37,38}$ The method we employed involved two general steps as implemented in the universal cluster-expansion software package. ${ }^{39}$ (Alternatively, the packages CLUPAN and ATAT could be used to the same effect. See, for example, Refs. 40-42.) First, a set of figures (correlation functions) was selected from a large pool generated from the parent lattice. The set of figures is typically smaller than the input data set and may be no larger if the system is to be well defined. Second, least-squares regression was used to determine the ECIs corresponding to these figures, given the input data. In short, the figure pool comprised the sample space from which many candidate fits were generated.

The fits were then scored using leave one out cross validation (LOOCV). Given $n$ input structures, LOOCV provides a quantitative measure of the quality of fit by averaging the error found using $n-1$ structures (training data) to predict 1 structure (validation data) for each of the $n$ structures,

$$
S_{\mathrm{cv}}=\frac{1}{n} \sum_{i=1}^{n}\left|E_{\mathrm{CE}}\left(\sigma_{n}\right)-E_{\mathrm{DFT}}\left(\sigma_{n}\right)\right| .
$$

The cross validation score, in units of energy, should be a small fraction of the energies of input structures so that reliable results are obtained. Typical fitting errors for the $\mathrm{Mg}-\mathrm{Li}$ system were between 0 and $3 \mathrm{meV} / \mathrm{atom}$. With input energies near $-30 \mathrm{meV} /$ atom, the errors were in the reasonable range. A total of 83 and 73 structures comprised the input data set used in the construction of bcc and hcp CEs, respectively.

Once a CE was constructed, a ground-state search was performed over large numbers of derivative superstructures (see Fig. 3). Thermodynamically stable ground states, how- 

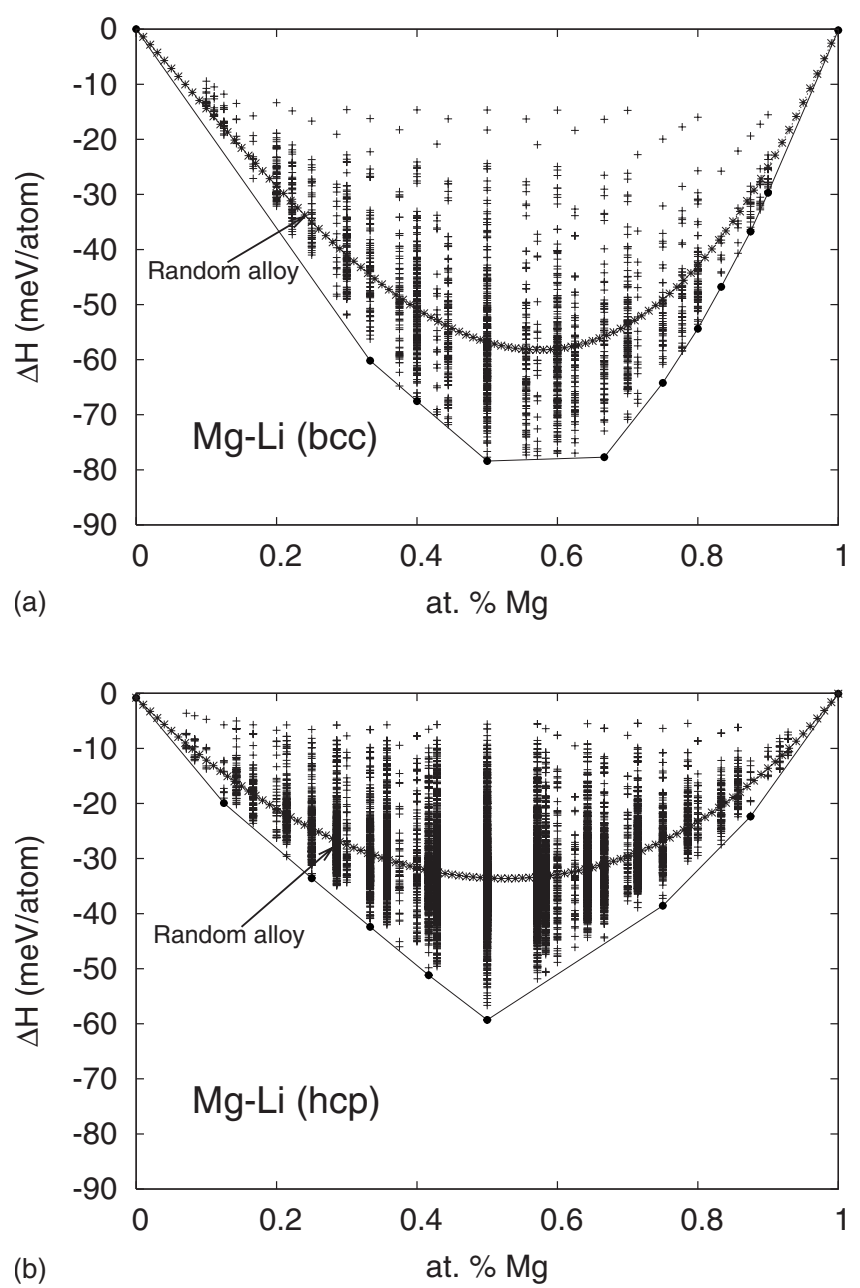

FIG. 3. Formation enthalpy versus concentration for many derivative superstructures. The random-alloy energy is included as well as the convex hull. Points on the convex hull indicate thermodynamically stable ground-state structures.

ever, are not necessarily the minimum-energy configurations at each concentration. One usually encounters a concentration in which the average energy of two structures with neighboring concentrations yields a mixed phase with lower total energy. The tie lines connecting all pairs of minimumenergy structures were thus determined. When the structure in question rests below all such tie lines it was included as a ground state. The complete set of structures determined in this manner comprises the minimal convex set of structures (the convex hull). Figure 3 includes the convex hulls for the bcc and hcp Mg-Li CE constructed using the predicted energies from fits generated using 83 and 73 input structures, respectively.

The absolute minima within the set of configurations that included both bcc and hcp superstructures were found by scaling hcp structure formation enthalpy by the difference in free energy of pure $\mathrm{Mg}$ and $\mathrm{Li}$ according to concentration. The free energy was computed for hcp $\mathrm{Mg}$ and $\mathrm{Li}$ and bcc $\mathrm{Mg}$ and $\mathrm{Li}$, and the formation enthalpy of hcp structures were adjusted in the following manner:

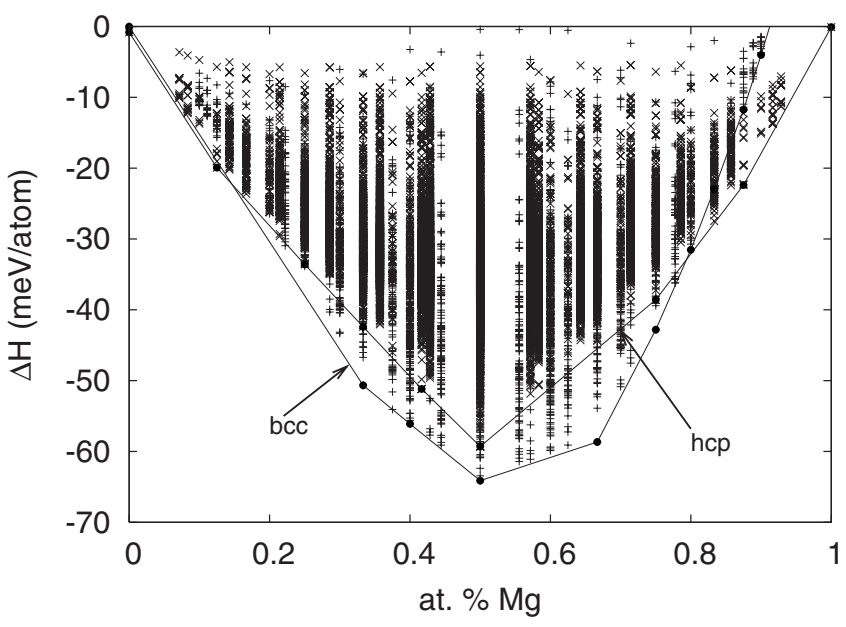

FIG. 4. CE formation enthalpies as a function of concentration. Energy predictions from the hcp CE are shifted according to the difference in free energy of $\mathrm{Mg}$ and $\mathrm{Li}$ on bcc and hcp lattices. Note the two-phase region of bcc and hcp phases on the Mg-rich side consistent with known phase data (Fig. 2).

$$
H \rightarrow H+\left[x \Delta H_{\mathrm{Mg}}+(1-x) \Delta H_{\mathrm{Li}}\right]
$$

Here $H$ is the formation enthalpy of a ground-state structure, $x$ is the concentration of $\mathrm{Mg}$, and $\Delta H_{\mathrm{Mg}}$ and $\Delta H_{\mathrm{Li}}$ are the differences in the hcp and bcc free energies of $\mathrm{Mg}$ and $\mathrm{Li}$. CE-predicted energies shown in Fig. 3 scaled relative to one another in this fashion are shown in Fig. 4.

In the case of bcc $\mathrm{Mg}-\mathrm{Li}$, the convex-hull-constructed using DFT energies of CE-predicted ground states reveal ground states $\mathrm{C} 11_{b}, \mathrm{~B} 2$, and $\mathrm{L}_{0}$ (see the Appendix for details of the method used). These structure types are in agreement with the HT results and are referred to by their Strukturbericht designation. It is perhaps of interest to note that the ground-state structures are several millielectron volt lower than the structure nearest in energy with the same concentration except at $50 \%$ (B2). In the case of B2, the energy is found to be only slightly lower than an $A_{2} B_{2}$ structure with [011] directed stacking planes (similar to the NbP facecentered-cubic superstructure) (Fig. 5). A slight difference in formation enthalpy is evidently the reason many CEs were unable to accurately predict the relative energies of this $\mathrm{NbP}$ like structure (corresponding to structure 19 in Table I) and the true ground state, B2.

$\mathrm{Mg}-\mathrm{Li}$ ground-state predictions on the hcp lattice were treated in the same manner as the bcc structures, i.e., VASP was used to directly compute their energies from which the ground states were determined. Ground states were found at concentrations $x=1 / 8,1 / 2,2 / 3$, and $3 / 4 \mathrm{Mg}$. In this case, the configurations do not correspond to known structures. Hcp phases were higher in energy than the bcc ground states across the concentration range excluding pure $\mathrm{Mg}$ (see Fig. 6 ). From the superposed energies of bcc and hcp Mg-Li, it can be seen that the two-phase region that extends from approximately 17 at. \% $\mathrm{Li}$ at finite temperatures broadens as the temperature is lowered and the solubility of $\mathrm{Mg}$ in $\mathrm{Li}$ decreases. 

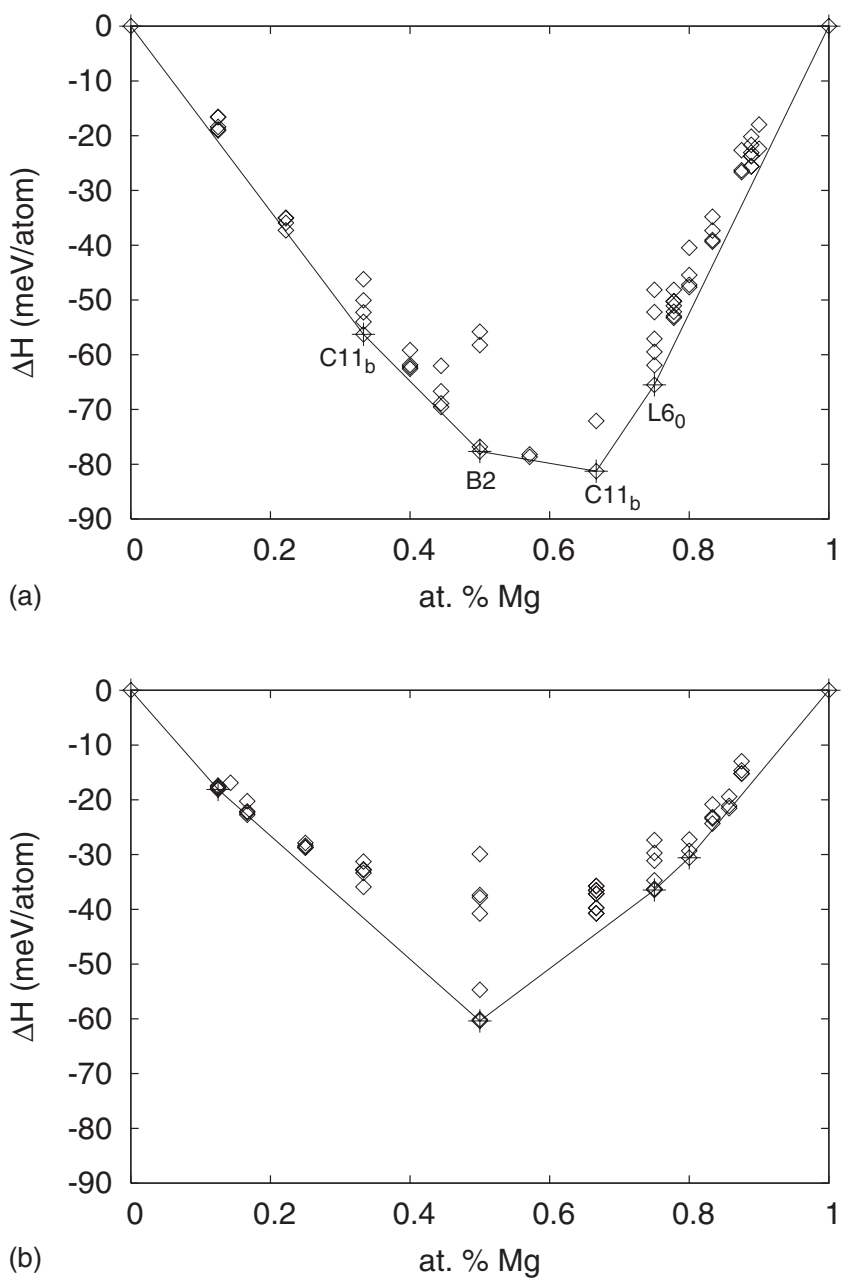

FIG. 5. Final ground-state predictions for bcc and hep Mg-Li using energies computed with VASP. The tie lines are shown. The hcp ground states do not correspond to commonly known structure types.

\section{ORDER-DISORDER TRANSITIONS PREDICTED BY MONTE CARLO SIMULATION}

Complex Ising-type Hamiltonians with many couplings are notoriously difficult to extend to finite temperatures with Monte Carlo (MC) methods, especially in the lowtemperature region. ${ }^{43}$ Numerical problems also arise when one attempts to model the system around the critical temperature (i.e., due to critical slowing). In general, the behavior of systems containing many interactions is not well understood and MC convergence times often make the task of modeling the system nearly intractable.

The CEs we constructed for Mg-Li utilized large numbers of figures in order to provide accurate energy predictions across the concentration range. As a result, however, Monte Carlo simulations involving these complex Hamiltonians present significant numerical difficulties. If determining the critical temperature is to be computationally feasible, it is necessary to simplify the complex $\mathrm{CE}$ to one that involves fewer interactions.
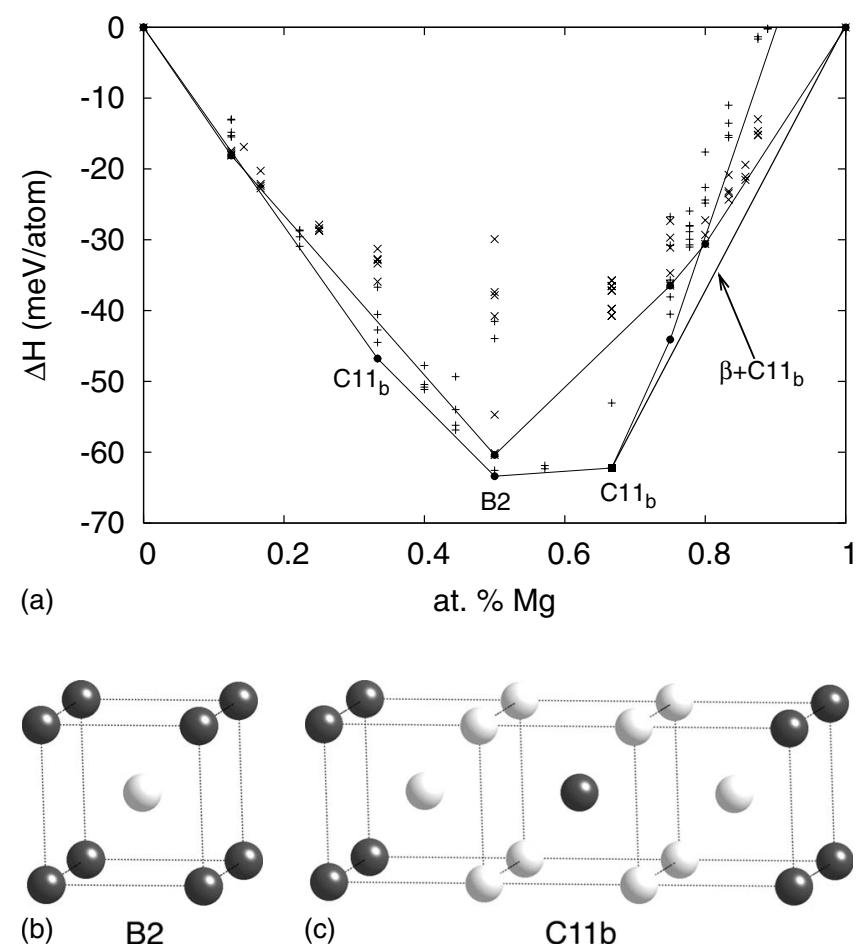

FIG. 6. Final ground-state predictions for hcp and bcc Mg-Li. The hcp energies are shifted relative to bcc energies. Note that there is no hcp phase predicted other than pure $\mathrm{Mg}$ and the two-phase region extending to $2 / 3 \mathrm{Mg}$. The structures $\mathrm{B} 2$ and $\mathrm{C} 11 \mathrm{~b}$ are also shown from left to right.

Although the simplified CE is certainly incapable of predicting the behavior of ordering as accurately as one containing many figures, by ensuring the proper ground states and energies are predicted, sufficiently accurate conclusions may be made. Further, rather than a detailed study of the nature of the transition, it was our primary interest to determine generally where the order-disorder transitions occur in order to guide experimental attempts to realize the ordered phases.

For the above reasons, a five-term $\mathrm{CE}$ on the bcc lattice was constructed for the purposes of conducting finitetemperature MC simulations. The statistical analysis conducted on many CEs provided the criteria by which we were able to narrow the selection of between many five-term CEs. Specifically it was required that the five-term CE-predicted ground states at $1 / 3,1 / 2$, and $2 / 3$ magnesium with structure types, $\mathrm{C} 11_{b}, \mathrm{~B} 2$, and $\mathrm{C} 11_{b}$, respectively, and that predicted energies were comparable to those computed using firstprinciples methods. In the five-term $\mathrm{CE}$, predicted energies differed from DFT calculations on average less than $5 \mathrm{meV}$ (roughly an order of magnitude smaller than input energies). Canonical Monte Carlo simulations were performed using the five-term $\mathrm{CE}$ on a $30 \times 30 \times 30$ bcc lattice with periodic boundary conditions. Equilibration times were between $5 \times 10^{6}$ and $15 \times 10^{6} \mathrm{MC}$ steps. The temperature was decreased uniformly in decrements of $10 \mathrm{~K}$ initially from 2000 to $50 \mathrm{~K}$. Transitions were identified by calculating the specific heat, using the usual statistical relation involving the mean-square deviation of the energy from the mean value, ${ }^{43}$ 

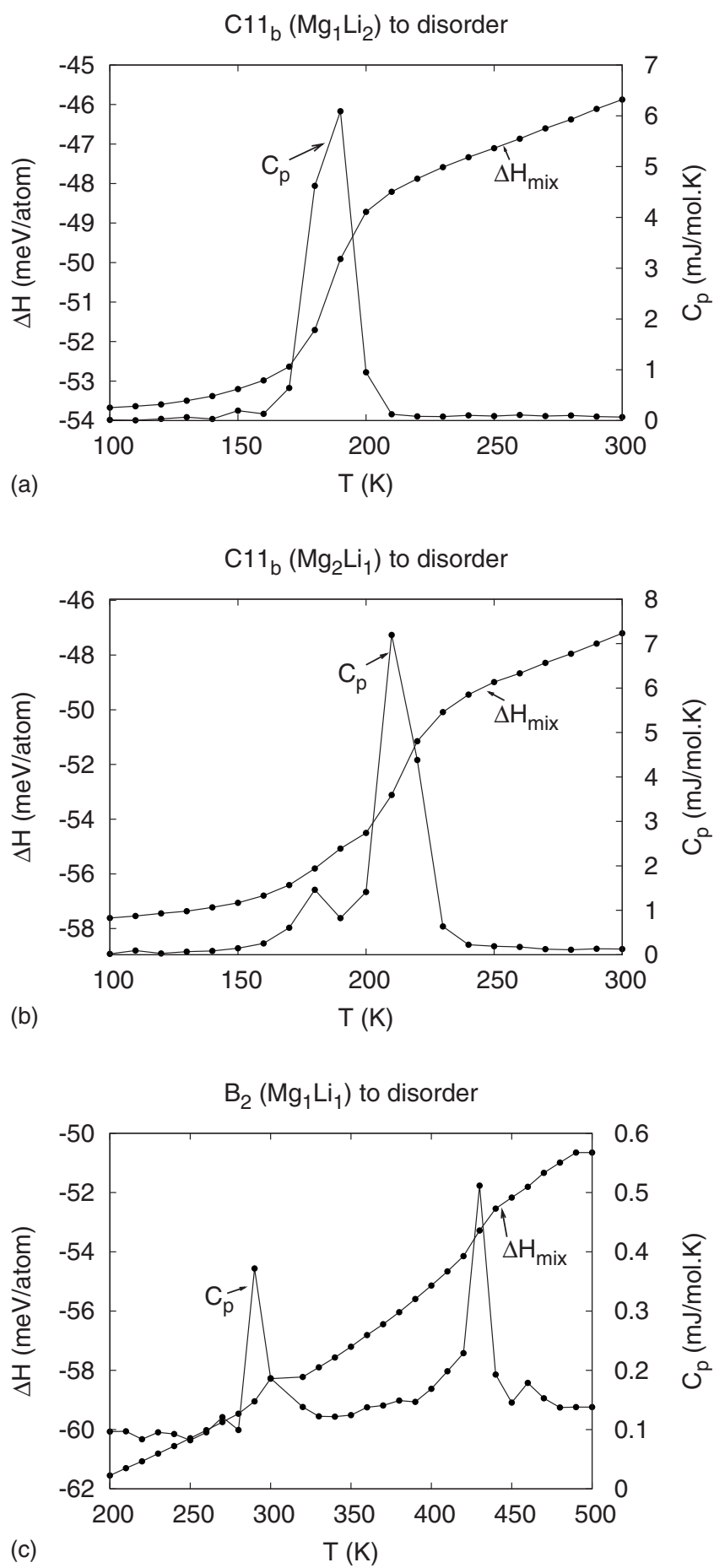

FIG. 7. Specific heat $C_{p}$ of Mg-Li ordered phases with temperature increased in increments of $10 \mathrm{~K}$. Formation enthalpy is overlaid including the scale indicated on the left.

$$
\sigma_{E}^{2}=\left\langle E^{2}\right\rangle-\langle E\rangle^{2}=\frac{\mathrm{C}}{k \beta^{2}} .
$$

Figure 7 displays the configurational specific heat of the three predicted ordered phases.

Transition temperatures were estimated by identifying the "divergence" in the heat capacity; the structure types $\mathrm{C} 11_{b}$ exhibit transitions at approximately 190 and $210 \mathrm{~K}$ for $1 / 3$ and 2/3 Mg, respectively. Simulations at 50\%, B2, exhibited more resistance to equilibration and required many more flips per MC time. Nevertheless, the transition was identified to be between 300 and $450 \mathrm{~K}$ by pair-correlation values. Comparison of pair-correlation values of the $\mathrm{MC}$ cell with the CE-predicted ground-state structures reveals good agreement after the transition. At 50\% the correlation values approach those of the expected B2 structure gradually over the temperature region shown (Fig. 7), suggesting that although the system was not fully equilibrated an order-disorder transition had occurred.

The range of transition temperatures predicted by the MC simulations is generally supported by the results of sonic measurements for low-temperature $\mathrm{Mg}$-Li alloys. In the work of Barrett and Trautz, ${ }^{12}$ the onset of spontaneous structural transitions were measured by recording audible clicks, the result of the rapid shearing motions that accompany the transition. For materials containing a large percentage of $\mathrm{Mg}$ content, few audible clicks were heard, and the specific nature of the partial ordering was not identifiable by diffraction techniques. However, the onset of spontaneous ordering for alloys containing greater than 20 at. \%. Mg were determined by noting the temperature at which the first clicks were heard and was estimated between 140 and $200 \mathrm{~K}$.

\section{CONCLUSION}

In conclusion, the $\mathrm{CE}$ method predicts bcc ordered phases at several concentrations including one which may be well suited for structural applications, $\mathrm{C} 11_{b}$. With the exception of pure $\mathrm{Mg}$, hcp phases were found to be higher in energy than bcc ground states across the concentration range. Orderdisorder transition temperatures, predicted by MC simulations, have been reported for the three bcc ordered structures between 200 and $400 \mathrm{~K}$.

The predicted low-temperature transitions of the $\mathrm{Mg}-\mathrm{Li}$ bcc ordered phases possibly explain why they have evaded experimental observation despite a relatively large amount of investigation in the system. Additionally, developing methods to experimentally realize the $\mathrm{Mg}-\mathrm{Li}$ ordered phases is likely to require significant effort due, in particular, to the low temperatures of the transitions and possible kinetic limitations.

However, the bulk low-temperature phases should not be ruled out, even at high temperature. Size-induced stabilization present at the nanoscale ${ }^{44,45}$ may promote stability at higher temperature for nanodispersed precipitates. Although the mechanical properties would not modify the solidsolution $\mathrm{Mg}$-Li appreciably, the overall effect in thermal and electric transport can be dramatic, as it has been shown for nanoprecipitates in semiconducting materials. ${ }^{46-48}$ Thus, the enhanced structural properties of alloys containing even small amounts of ordering provide a significant incentive in this regard. Furthermore, low-temperature ordering has the inherent advantage of increased stability at higher temperatures possibly allowing for practical application of the ordered alloys if initially realized. 


\section{ACKNOWLEDGMENTS}

R.H. Taylor and G.L.W. Hart are grateful for the financial support provided by the NSF under Grants No. DMR0650406 and No. DMR-0908753. S. Curtarolo acknowledges support by ONR (Grants No. N00014-07-1-0878, No. N00014-07-1-1085, and No. N00014-09-1-0921) and NSF (Grants No. DMR-0639822 and No. DMR-0650406). The Fulton Supercomputing Center at Brigham Young University generously provided computing time and technical support for this project. The authors would also like to thank Didier de Fontaine, Ohad Levy, Mike Mehl, Leeor Kronik, and Wahyu Setyawan for fruitful discussions and helpful comments. S.C. acknowledges support from the Weizmann Institute of Science.

\section{APPENDIX}

The genetic algorithm and leave one out cross validation provide a useful framework for constructing a $\mathrm{CE}$ that accurately fits input data; furthermore, a CE produced in this manner is capable of predicting the energy of structures not included in the input set provided that the "physics" of the input data is representative of the entire system (although off-lattice structures are not included in the configuration space, HT and experimental data suggest that additional structures will not form in Mg-Li. Not only are the HT energies higher than both hcp and bcc CE-predicted structure energies but the majority of HT ground-state predictions are bcc superstructures.)

By adding structures in underfit regions to the input structure set, the overall predictive capacity of the CE was improved. Low-energy structures without similar input structures as well as additional structures similar in configuration to poorly predicted input structures were added to the input set. Structures were added in this manner by comparing successive fits so that the final input data set was determined to ensure a broadly accurate parameterization of configuration space. ${ }^{37}$ A total of 85 bcc-derived and 71 hcp-derived superstructures were ultimately used in the construction of the CEs.

The utility of the iterative process hints at the underlying physical workings of the CE method. Input structures contribute to the CE via the addition of physics yet unaccounted for. Iteratively adding structures from regions of poorly fit data, and from regions lacking input data, will then, in principle, lead to a CE capable of making accurate predictions across configuration space. Furthermore, given a set if interaction terms representative of the system, the inclusion of additional input data should do little to alter the ground-state predictions of the $\mathrm{CE}$ constructed using these figures.

The durability of the fit, i.e., the immutability of groundstate predictions with the inclusion of new input data, was studied in the following manner. A database containing the complete set of input structures was parsed into input sets of increasing size; this was done several times with unique parsing, and a CE was constructed for each input set. Ground-state predictions made by the CEs were compared for agreement, and frequently predicted ground-state concentrations formed a group of credible predictions.
TABLE I. Columns A-E contain the predicted structures (numbered in accordance with the enumeration algorithm) from five 85input generated fits for bcc Mg-Li. The percentage occurrence of ground states to the total number of fits (195) is given in the second column. Except for $9 / 10 \%$ and $2 / 9 \% \mathrm{Mg}$ ground states, the predictions in A-E affirm the ground-state predictions made by the majority of total fits (195).

\begin{tabular}{rrrrrrr}
\hline \hline$x(\mathrm{Mg})$ & $\begin{array}{r}\text { No. of occurrences/195 } \\
(\%)\end{array}$ & $\mathrm{A}$ & $\mathrm{B}$ & $\mathrm{C}$ & $\mathrm{D}$ & $\mathrm{E}$ \\
\hline $1 / 8$ & 24 & 631 & 631 & & & \\
$2 / 9$ & 11 & 1134 & & 1134 & & 1134 \\
$1 / 3$ & 66 & 10 & 125 & 10 & 10 & 10 \\
$3 / 8$ & 27 & & & & & 404 \\
$2 / 5$ & 59 & 45 & 45 & 45 & 45 & 48 \\
$4 / 9$ & 21 & 938 & & & & \\
$1 / 2$ & 97 & 19 & 19 & 19 & 19 & 19 \\
$4 / 7$ & 27 & & & 191 & 191 & \\
$2 / 3$ & 92 & 7 & 7 & 7 & 7 & 7 \\
$3 / 4$ & 70 & 277 & 277 & 277 & 277 & 277 \\
$7 / 9$ & 32 & & 1112 & & 1112 & \\
$4 / 5$ & 70 & 54 & & 54 & & 54 \\
$5 / 6$ & 18 & & 62 & & & \\
$7 / 8$ & 52 & & 593 & 593 & 593 & 436 \\
$8 / 9$ & 63 & 1052 & & & & \\
$9 / 10$ & 31 & & 1145 & 1142 & & 2309 \\
\hline \hline
\end{tabular}

For bec $\mathrm{Mg}-\mathrm{Li}$, a database containing the formation enthalpies of 85 input structures were parsed into 39 groups of input structures five times. CEs were constructed for each of the 39 groups yielding 39 predictions of the $\mathrm{Mg}-\mathrm{Li}$ ground states. The structures and associated concentrations predicted by fits containing the entirety of the input data $(\mathrm{A}-\mathrm{E})$ are listed in Table I. The frequencies of prediction of ground states at each listed concentration are shown in the second

TABLE II. Hcp Mg-Li ground-state predictions as in Table I. Several frequent predictions are not affirmed by full fits and thus additional concentrations have been included.

\begin{tabular}{|c|c|c|c|c|c|c|}
\hline$x(\mathrm{Mg})$ & $\begin{array}{c}\text { No. of occurrences/165 } \\
(\%)\end{array}$ & A & B & $\mathrm{C}$ & D & $\mathrm{E}$ \\
\hline $1 / 8$ & 33 & 333 & & 333 & 318 & 333 \\
\hline $1 / 7$ & 36 & & & & & \\
\hline $1 / 6$ & 72 & 3920 & 3920 & 3920 & 3920 & 3920 \\
\hline $1 / 4$ & 83 & & & & & \\
\hline $1 / 3$ & 37 & & & & & \\
\hline $1 / 2$ & 100 & 12 & 12 & 12 & & 12 \\
\hline $2 / 3$ & 64 & & & & & \\
\hline $3 / 4$ & 61 & 321 & 321 & 321 & & 303 \\
\hline $4 / 5$ & 30 & & & & & \\
\hline $5 / 6$ & 82 & 1491 & 1491 & 1491 & 1491 & 1491 \\
\hline $6 / 7$ & 38 & & & & & \\
\hline $7 / 8$ & 18 & & 319 & & & \\
\hline
\end{tabular}


column. It is seen that the predictions made by CEs constructed using the entire input set generally correspond to predictions made by the majority of fits.

The hcp Mg-Li system was treated similarly. Input data computed for 71 input structures was parsed into 33 unique input sets five times. In contrast to the bcc case, however, several concentrations not predicted by CEs constructed using the complete input data were found to have a high frequency of prediction by the totality of fits. Thus, these are also included in the table (Table II).
The potential ground-state pool was significantly reduced by comparing the predictions of many CEs. However, because the precision necessary to distinguish small differences in structure energetics are not to be expected from the finite CE, DFT was used to directly compare the energies of the final group of candidates. VASP was used to evaluate each of the candidate structures listed in Tables I and II as well as candidate structures not necessarily predicted by fits A-E but with significant frequency of occurrence.
*Corresponding author; gus.hart@gmail.com

${ }^{1}$ G. Shen and B. Duggan, Metall. Mater. Trans. A 38, 2593 (2007).

${ }^{2}$ R. Jackson and P. Frost, NASA Report No. SP-5068, 1967 (unpublished).

${ }^{3}$ P. D. Frost, NASA Report No. SP-5028, 1965 (unpublished).

${ }^{4}$ W. Counts, M. Friák, D. Raabe, and J. Neugebauer, Acta Mater. 57, 69 (2009).

${ }^{5}$ J. Catterall, Nature (London) 169, 336 (1952).

${ }^{6}$ G. Grube, H. Zeppelin, and H. Bumm, Z. Elektrochem. 40, 160 (1934).

${ }^{7}$ P. Saldau and F. Schamray, Z. Anorg. Chem. 224, 388 (1935).

${ }^{8}$ W. Freeth and G. Raynor, J. Inst. Met. 82, 575 (1953).

${ }^{9}$ W. Hume-Rothery, G. Raynor, and E. Butchers, J. Inst. Met. 71, 589 (1945)

${ }^{10}$ W. Gasior, Z. Moser, W. Zakulski, and G. Schwitzgebel, Metall. Mater. Trans. A 27, 2419 (1996).

${ }^{11}$ A. Nayeb-Hashemi, J. Clarck, and A. Pelton, Bull. Alloy Phase Diagrams 5, 365 (1984).

${ }^{12}$ C. Barrett and O. Trautz, Trans. Metall. Soc. AIME 175, 579 (1948).

${ }^{13}$ R. Berry and G. Raynor, Nature (London) 171, 1078 (1953).

${ }^{14}$ F. Herbstein and B. Averbach, Acta Crystallogr. 9, 91 (1956).

${ }^{15}$ S. Curtarolo, D. Morgan, and G. Ceder, CALPHAD: Comput. Coupling Phase Diagrams Thermochem. 29, 163 (2005).

${ }^{16}$ The GSS results presented here were performed over $16098 \mathrm{bcc}$ and 15975 hcp derived superstructures. Searches including many more structures were also performed but yielded no new ground states.

${ }^{17}$ A. Nayeb-Hashemi, J. Clarck, and A. Pelton, Binary Alloy Phase Diagrams, 2nd ed. (Materials International Society, Materials Park, OH, 1990), Vol. 3.

${ }^{18}$ N. Saunders, CALPHAD: Comput. Coupling Phase Diagrams Thermochem. 14, 61 (1990).

${ }^{19}$ E. Schürmann and H.-J. Voss, Giessereiforschung 33 (1), 33 (1981).

${ }^{20}$ M.-L. Saboungi and C. C. Hsu, CALPHAD: Comput. Coupling Phase Diagrams Thermochem. 1, 237 (1977).

${ }^{21}$ O. Henry and H. Cordiano, Trans. Am. Inst. Min., Metall. Pet. Eng. 111, 319 (1934).

${ }^{22}$ G. Kresse and J. Hafner, Phys. Rev. B 47, 558 (1993).

${ }^{23}$ S. Froyen, Phys. Rev. B 39, 3168 (1989).

${ }^{24}$ G. Kresse and D. Joubert, Phys. Rev. B 59, 1758 (1999).

${ }^{25}$ P. E. Blöchl, Phys. Rev. B 50, 17953 (1994).

${ }^{26}$ S. Curtarolo, D. Morgan, K. Persson, J. Rodgers, and G. Ceder,
Phys. Rev. Lett. 91, 135503 (2003).

${ }^{27}$ D. Morgan, G. Ceder, and S. Curtarolo, Meas. Sci. Technol. 16, 296 (2005).

${ }^{28}$ O. Levy, R. V. Chepulskii, G. L. W. Hart, and S. Curtarolo, J. Am. Chem. Soc. 132, 833 (2010).

${ }^{29}$ S. Curtarolo, G. L. W. Hart, W. Setyawan, R. V. Chepulskii, O. Levy, and D. Morgan, "AFLOW: software for high-throughput calculation of material properties," http://materials.duke.edu/ aflow.html (2009).

${ }^{30}$ G. L. W. Hart and R. W. Forcade, Phys. Rev. B 77, 224115 (2008).

${ }^{31}$ G. L. W. Hart and R. W. Forcade, Phys. Rev. B 80, 014120 (2009).

${ }^{32} \mathrm{~A} 5, \mathrm{~A} 6, \mathrm{~A} 7, \mathrm{~A} 8, \mathrm{~A} 9, \mathrm{~A} 11, \mathrm{~B} 20, \mathrm{C} 36, \mathrm{D} 5_{19}, \mathrm{Al}_{2} \mathrm{Zr}_{4}, \mathrm{Al}_{3} \mathrm{Zr}_{2}, \mathrm{CdTi}$, $\mathrm{CuPt}_{7}, \mathrm{Cu}_{3} \mathrm{Ti}_{2}, \mathrm{Ga}_{2} \mathrm{Hf}, \mathrm{Ga}_{4} \mathrm{Ni}, \mathrm{Ga}_{3} \mathrm{Pt}_{5}, \mathrm{Ga}_{4} \mathrm{Ti}_{5}, \mathrm{Hg}_{2} \mathrm{Pt}$, ITl, InTh, LiB-MS1/2 (Ref. 49), $\mathrm{NbNi}_{8}, \mathrm{NiTi}_{2}, \mathrm{SeTl}$, and $\mathrm{V}_{4} \mathrm{Zn}_{5}$.

${ }^{33}$ P. Villars, M. Berndt, K. Brandenburg, K. Cenzual, J. Daams, F. Hulliger, T. Massalski, H. Okamoto, K. Osaki, A. Prince, H. Putz, and S. Iwata, J. Alloys Compd. 367, 293 (2004).

${ }^{34}$ Binary Alloy Phase Diagrams, edited by T. B. Massalski, H. Okamoto, P. R. Subramanian, and L. Kacprzak (American Society for Metals, Metals Park, OH, 1990).

${ }^{35}$ It should also be noted that systems exist which do not conserve the parents' lattice [i.e., Hf-Ti, Hf-Zr (Ref. 50)].

${ }^{36}$ J. Sanchez, F. Ducastelle, and D. Gratias, Physica A 128, 334 (1984).

${ }^{37}$ G. L. W. Hart, V. Blum, M. J. Walorski, and A. Zunger, Nature Mater. 4, 391 (2005).

${ }^{38}$ V. Blum, G. L. W. Hart, M. J. Walorski, and A. Zunger, Phys. Rev. B 72, 165113 (2005).

${ }^{39}$ D. Lerch, O. Wieckhorst, G. L. W. Hart, R. W. Forcade, and S Muller, Modell. Simul. Mater. Sci. Eng. 17, 055003 (2009).

${ }^{40}$ A. van de Walle, M. Asta, and G. Ceder, CALPHAD: Comput. Coupling Phase Diagrams Thermochem. 26, 539 (2002).

${ }^{41}$ A. van de Walle, Nature Mater. 7, 455 (2008).

${ }^{42}$ A. Seko, Y. Koyama, and I. Tanaka, Phys. Rev. B 80, 165122 (2009).

${ }^{43}$ D. P. Landau and K. Binder, A Guide to Monte Carlo Simulations in Statistical Physics (Cambridge University Press, Cambridge, UK, 2005)

${ }^{44}$ A. R. Harutyunyan, N. Awasthi, A. Jiang, W. Setyawan, E. Mora, T. Tokune, K. Bolton, and S. Curtarolo, Phys. Rev. Lett. 100, 195502 (2008).

${ }^{45}$ S. Curtarolo, N. Awasthi, W. Setyawan, A. Jiang, K. Bolton, T. Tokune, and A. R. Harutyunyan, Phys. Rev. B 78, 054105 
(2008).

${ }^{46}$ W. Kim, J. Zide, A. Gossard, D. Klenov, S. Stemmer, A. Shakouri, and A. Majumdar, Phys. Rev. Lett. 96, 045901 (2006).

${ }^{47}$ S. Wang and N. Mingo, Appl. Phys. Lett. 94, 203109 (2009).

${ }^{48}$ N. Mingo, D. Hauser, N. P. Kobayashi, M. Plissonnier, and A.
Shakouri, Nano Lett. 9, 711 (2009).

${ }^{49}$ A. N. Kolmogorov and S. Curtarolo, Phys. Rev. B 73, 180501(R) (2006); 74, 224507 (2006).

${ }^{50}$ O. Levy, G. L. W. Hart, and S. Curtarolo, Acta Mater. (to be published). 